\title{
Use of transcranial direct current stimulation for the treatment of auditory hallucinations of schizophrenia - a systematic review
}

This article was published in the following Dove Press journal:

Neuropsychiatric Disease and Treatment

I February 2017

Number of times this article has been viewed

\author{
Pedro H Pondé,' \\ Eduardo P de Sena, ${ }^{2}$ Joan \\ A Camprodon, ${ }^{3}$ Arão \\ Nogueira de Araújo, ${ }^{2}$ Mário \\ F Neto, ${ }^{4}$ Melany DiBiasi, ${ }^{5}$ \\ Abrahão Fontes Baptista, ${ }^{6,7}$ \\ Lidia MVR Moura, ${ }^{8}$ Camila \\ Cosmo $2,3,6,9,10$
}

'Dynamics of Neuromusculoskeletal System Laboratory, Bahiana School of Medicine and Public Health, ${ }^{2}$ Postgraduate Program in Interactive Process of Organs and Systems, Federal University of Bahia, Salvador, Bahia, Brazil; ${ }^{3}$ Laboratory for Neuropsychiatry and Neuromodulation and Transcranial Magnetic Stimulation Clinical Service, Department of Psychiatry, Massachusetts General Hospital, Harvard Medical School, Boston, MA, USA; ${ }^{4}$ Scientific Training Center Department, School of Medicine of Bahia, Federal University of Bahia, Salvador, Bahia, Brazil; ${ }^{5}$ Neuromodulation Center, Spaulding Rehabilitation Hospital, Harvard Medical School, Boston, MA, USA; ${ }^{6}$ Functional Electrostimulation Laboratory, Biomorphology Department, ${ }^{7}$ Postgraduate Program on Medicine and Human Health, School of Medicine, Federal University of Bahia, Salvador, Bahia, Brazil; ${ }^{8}$ Department of Neurology, Massachusetts General Hospital, Harvard Medical School, Boston, MA, USA; ${ }^{9}$ Center for Technological Innovation in Rehabilitation, Federa University of Bahia, ${ }^{10}$ Bahia State Health Department (SESAB), Salvador, Bahia, Brazi

Correspondence: Camila Cosmo Institute of Health Sciences, Federal University of Bahia, Avenida Reitor Miguel Calmon, s/n, Vale do Canela, Salvador, Bahia 40110-100, Brazil

Tel +55 7l 32838885

Email ccosmo@ufba.br
Introduction: Auditory hallucinations are defined as experiences of auditory perceptions in the absence of a provoking external stimulus. They are the most prevalent symptoms of schizophrenia with high capacity for chronicity and refractoriness during the course of disease. The transcranial direct current stimulation (tDCS) - a safe, portable, and inexpensive neuromodulation technique has emerged as a promising treatment for the management of auditory hallucinations.

Objective: The aim of this study is to analyze the level of evidence in the literature available for the use of tDCS as a treatment for auditory hallucinations in schizophrenia.

Methods: A systematic review was performed, searching in the main electronic databases including the Cochrane Library and MEDLINE/PubMed. The searches were performed by combining descriptors, applying terms of the Medical Subject Headings (MeSH) of Descriptors of Health Sciences and descriptors contractions. PRISMA protocol was used as a guide and the terms used were the clinical outcomes ("Schizophrenia" OR "Auditory Hallucinations" OR "Auditory Verbal Hallucinations" OR "Psychosis") searched together (“AND”) with interventions ("transcranial Direct Current Stimulation" OR "tDCS" OR "Brain Polarization").

Results: Six randomized controlled trials that evaluated the effects of tDCS on the severity of auditory hallucinations in schizophrenic patients were selected. Analysis of the clinical results of these studies pointed toward incongruence in the information with regard to the therapeutic use of tDCS with a view to reducing the severity of auditory hallucinations in schizophrenia. Only three studies revealed a therapeutic benefit, manifested by reductions in severity and frequency of auditory verbal hallucinations in schizophrenic patients.

Conclusion: Although tDCS has shown promising results in reducing the severity of auditory hallucinations in schizophrenic patients, this technique cannot yet be used as a therapeutic alternative due to lack of studies with large sample sizes that portray the positive effects that have been described.

Keywords: auditory hallucinations, tDCS, schizophrenia, non-invasive brain stimulation

\section{Introduction}

Schizophrenia is a chronic mental disturbance characterized by dysfunctions in emotion, cognition, and perception of reality. ${ }^{1}$ Due to the potential of affecting innumerable cerebral domains, schizophrenic patients present with a heterogeneity of symptoms and variation in their severity during the evolution of the disease. ${ }^{2}$ The estimated prevalence of this disease is $\sim 1 \%$ among the world population, and its incidence is $\sim 1.5 / 10,000$ that reveals the capacity for chronification of this disease. ${ }^{3,4}$

The habitual clinical expression of schizophrenia is the presence of psychotic (positive) and negative symptoms that may be associated with a cognitive compromise, 
which generally is subtle. ${ }^{5}$ So-called "positive symptoms" comprised hallucinations, delusions, disturbances of thought, and bizarre behavior, whereas the "negative symptoms" include emotional dullness, anhedonia, alogia, and attention deficit. ${ }^{1}$ Hallucinations are defined as experiences of perception that occur without the real presence of an external stimulus. ${ }^{6}$ Although they may occur in any of the sensory modalities, the most common type is auditory verbal hallucination (AVH). ${ }^{7,8}$ Around $60 \%-80 \%$ of schizophrenic patients are estimated to present with this symptom during the course of the disease, and the perception of voices that talk to, comment, or issue commands to the individual has been reported. ${ }^{8,9}$ The majority of these hallucinations has a negative content, generally expressed by angry voices that use words or expressions of abuse to transmit information to the subject, and is associated with a high risk of suicide in schizophrenia. ${ }^{10,11}$

Recent advances in functional neuroimaging methods have enabled a better understanding of the disturbances of the neural circuits of neuropsychiatric diseases. ${ }^{12}$ For this reason, a growing interest in interventions with potentials of neuromodulation has arisen with a view to rupturing patterns of neuronal activity that may be associated with symptoms of a base disease. ${ }^{13}$

In the present context, transcranial direct current stimulation (tDCS) configures as a tool that potentiates cognitive and motor performance in healthy individuals and as an emergent treatment for a series of neuropsychiatric diseases. ${ }^{12,13}$ tDCS acts through the application of direct electrical currents of low amplitude on the scalp, modulating the cortical excitability. ${ }^{14}$

tDCS long-term effects appear to be associated with neuroplasticity phenomena that promote the modulation of neuronal synapses. ${ }^{14}$ Changes in the efficacy of activating NMDA receptors, GABAergic activity, and modulation of interneurons appear to be the main factors responsible for changes in long-term potentiation and depression. ${ }^{15,16}$ Another type of synaptic neuroplasticity that appears to be influenced by tDCS is the type dependent on the brainderived neurotrophic factor, in which its dysfunction is associated with the maladaptive neuroplasticity phenomena present in various neuropsychiatric diseases, including schizophrenia. ${ }^{1,16,17}$ During AVH, it is supposed that cerebral areas involved in the perception of the discourse, primary auditory cortex and associated areas of language in the left hemisphere, are pathologically hyperactive. ${ }^{18,19}$

Our aim is to analyze the available literature and, from it, determine the level of evidence of information for suggesting directions for future research and the indications for using tDCS in the therapeutic approach to auditory hallucinations in schizophrenia.

\section{Methods}

A systematic review of the literature was performed based on "The Cochrane Collaboration" methodology and guided by the PRISMA protocol. Scientific articles that approached the use of tDCS for the treatment of auditory hallucinations resistant to treatment with antipsychotic drugs in schizophrenia were searched in the following electronic database sources: "MEDLINE/PubMed", "Web of Science", "LILACS", "BIREME", and "PsycINFO", using the combination of descriptors, including terms from the Medical Subject Headings (MeSH) of descriptors of health sciences and contractions of descriptors. Therefore, the following terms were combined using the Boolean operator "AND":

- Outcome: "Schizophrenia" OR "Auditory Hallucinations"

OR “Auditory Verbal Hallucinations" OR "Psychosis".

- Intervention: "Transcranial Direct Current Stimulation (tDCS)" OR “tDCS" OR "Brain Polarization".

The research was conducted in an equal manner among all the databases relating each outcome to a type of intervention. The results of these combinations during the search were as follows: [Schizophrenia OR Auditory Hallucinations OR Auditory Verbal Hallucinations OR Psychosis] AND [Transcranial Direct Current Stimulation OR tDCS OR Brain Polarization].

The inclusion criteria applied to the studies were (1) use of tDCS in schizophrenic patients; (2) application of clinical scales in evaluating the severity of auditory hallucinations pre/post tDCS; (3) methodological design based on randomization, containing mean and standard deviation of outcomes analyzed; or data that would make it possible to deduce these values; (4) studies published in indexed periodicals; and (5) publications in Portuguese, English, or Spanish.

The exclusion criteria used were (1) studies containing samples of schizophrenic patients presenting neuropsychiatric comorbidities; (2) review articles; (3) studies that presented another cerebral stimulation technique; and (4) studies identified, but without full version available.

Two investigators independently conducted the search for and selection of articles. The titles and abstracts of all publications found were imported to the reference manager EndNote $X 7$ that was responsible for eliminating the duplicates.

The titles and abstracts of studies found were read by each researcher for the purpose of finding potentially useful 


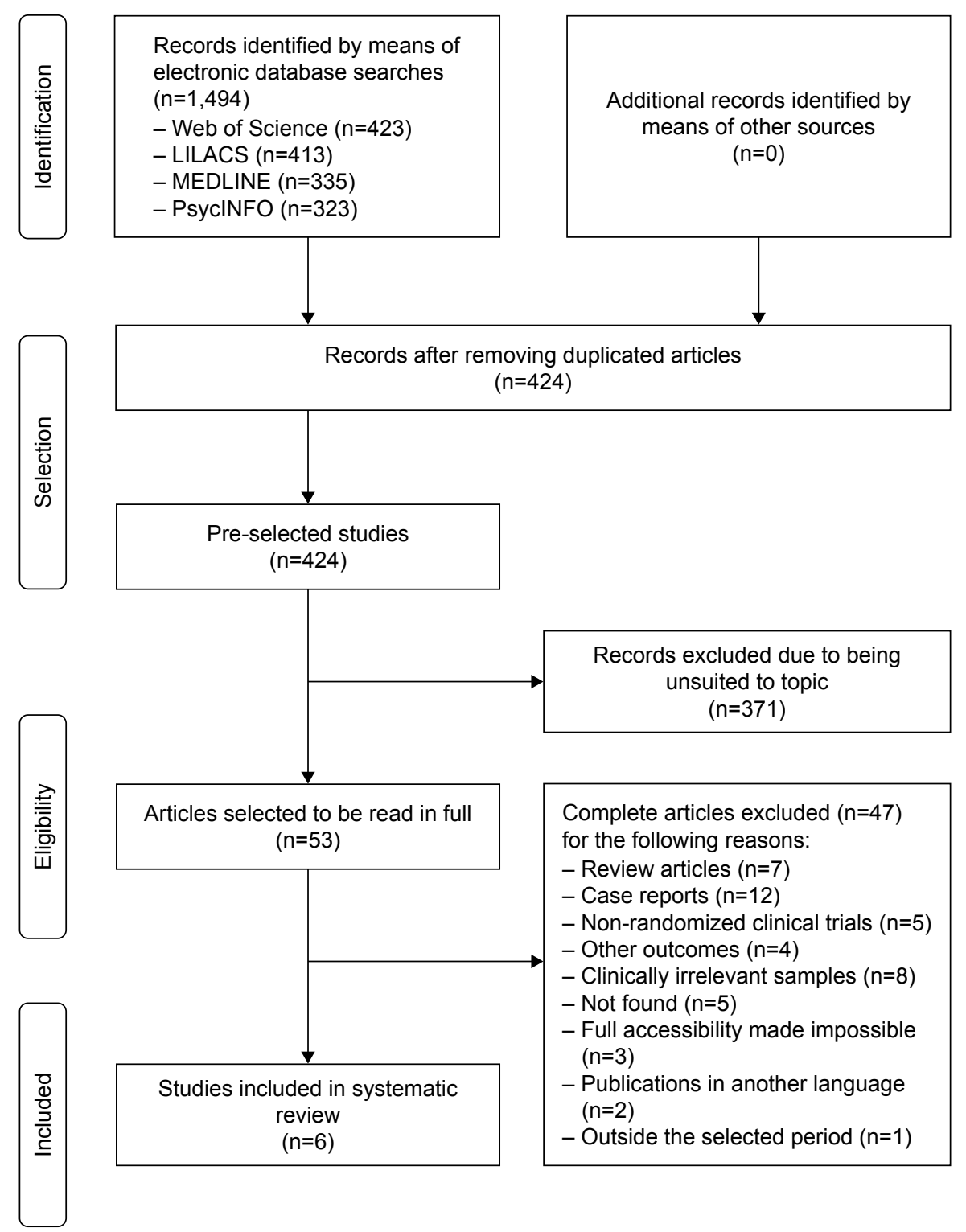

Figure I Flow diagram of selection process.

articles. Those that in principle fulfilled the selection criteria were read in full for better verification and then either included in this systematic review or not. The references of the articles found were consulted to seek new articles that may not have been found in the initial search.

Initially, 1,494 publications were identified by means of electronic search. The databases that resulted in findings were Web of Science ( $n=423)$; LILACS ( $n=413)$; MEDLINE/ PubMed ( $\mathrm{n}=335)$, and PsycINFO ( $\mathrm{n}=323)$. After eliminating duplicated articles, the titles and abstracts of 424 articles were read by both researchers (PHP, EPS), independently, totaling a number of 53 pre-selected manuscripts for reading in full. During this stage, another 371 articles were excluded because they were not suited to the topic.
Among the 53 articles initially selected, only 6 manuscripts fulfilled the eligibility criteria and were selected for final analysis and revision of their content, whereas the remainder were eliminated because they did not satisfy some of the pre-determined demands of the eligibility criteria (Figure 1). The manual search strategy was also applied to the lists of references of the articles found, but added no new studies to the final analysis.

The clinical and stimulation parameters adopted by each study are described in Tables 1 and 2, respectively.

\section{Results}

Brunelin et al conducted a randomized, parallel, doubleblind, sham-controlled tDCS trial placing the anode on the 
Table I Clinical parameters of studies obtained for systematic review

\begin{tabular}{|c|c|c|c|c|c|}
\hline References & $\mathbf{n}$ & Diagnosis & $\begin{array}{l}\text { Diagnostic } \\
\text { criteria }\end{array}$ & Eligibility criteria & $\begin{array}{l}\text { Outcome measurements } \\
\text { extracted }\end{array}$ \\
\hline Brunelin et $\mathrm{al}^{20}$ & 30 & Schizophrenia & DSM-IV & $\begin{array}{l}\text { Patients with AVH refractory to treatment with } \\
\text { antipsychotic drugs } \\
\text { Subjects with AVH and persistent negative symptoms } \\
\text { after failure of therapy with two different antipsychotic } \\
\text { agents with adequate posologies }\end{array}$ & AHRS \\
\hline Fitzgerald et $\mathrm{a}^{23}$ & 24 & $\begin{array}{l}\text { Schizophrenia and } \\
\text { schizoaffective disorder }\end{array}$ & M.I.N.I. & $\begin{array}{l}\text { Patients excluded were those presenting with } \\
\text { neuropsychiatric comorbidities and pregnant women } \\
\text { who used carbamazepine or who used antipsychotic } \\
\text { drugs irregularly }\end{array}$ & PANSS \\
\hline Mondino et $\mathrm{al}^{25}$ & 28 & Schizophrenia & Not related & Individuals with $\mathrm{AVH}$ refractory to treatment & Frequency of $\mathrm{AVH}$ \\
\hline Mondino et $\mathrm{al}^{26}$ & 23 & Schizophrenia & DSM-IV & $\begin{array}{l}\text { Patients who exhibited AVH daily, in spite of regular } \\
\text { use of antipsychotic drugs with adequate dose for a } \\
\text { minimum of } 3 \text { months. Patients excluded were those } \\
\text { with concomitant diagnosis of Axis I disorder }\end{array}$ & AHRS \\
\hline Smith et $\mathrm{al}^{27}$ & 33 & $\begin{array}{l}\text { Schizophrenia and } \\
\text { schizoaffective disorder }\end{array}$ & DSM-IV & $\begin{array}{l}\text { Regular smoker patients who lived in residences } \\
\text { in the study community }\end{array}$ & AHRS and PANSS \\
\hline Fröhlich et $\mathrm{a}^{28}$ & 26 & $\begin{array}{l}\text { Schizophrenia and } \\
\text { schizoaffective disorder }\end{array}$ & DSM-IV & $\begin{array}{l}\text { Subjects who presented at least } 3 \text { AVH episodes/week } \\
\text { for at least } 12 \text { weeks, with fixed antipsychotic posology } \\
\text { for a minimum of } 4 \text { weeks before the study began }\end{array}$ & AHRS \\
\hline
\end{tabular}

Abbreviations: AHRS, Auditory Hallucination Rating Scale, AVH, auditory verbal hallucinations; DSM-IV, Diagnostic and Statistical Manual of Mental Disorders-IV; M.I.N.I, Mini-Interview Neuropsychiatric Interview; n, number of patients included in the study; PANSS, Positive and Negative Syndrome Scale.

left dorsolateral prefrontal cortex (DLPFC) and cathode over left temporo-parietal junction (TPJ). ${ }^{20}$ Thirty DSM-IV schizophrenic patients with drug-resistant AVH (15 active and 15 sham) were submitted to twice daily stimulation sessions for 5 days (20 minutes; $2 \mathrm{~mA}$; electrode area of $35 \mathrm{~cm}^{2}$ ). The primary outcome was change in the severity of the AVH measured by the Auditory Hallucination Rating Scale (AHRS). ${ }^{21}$ The active group had a significant mean improvement of $31 \%$ and the therapeutic effects lasted for 3 months. There was also a significant improvement in negative symptoms as measured by the Positive and Negative Syndrome Scale (PANSS). ${ }^{22}$

In a randomized crossover trial, Fitzgerald et al investigated the therapeutic potential of unilateral and bilateral tDCS for the treatment of refractory AVH. ${ }^{23}$ Patients with schizophrenia or schizoaffective disorder presenting persistent hallucinations and negative symptoms, with failure to respond to two adequate therapeutic attempts with antipsychotic agents, were randomly allocated to bilateral tDCS (anode on F3/F4 and cathode over TP3/TP4; n=11)

Table 2 tDCS parameters of studies obtained for systematic review

\begin{tabular}{|c|c|c|c|c|c|c|c|}
\hline References & tDCS device & $\begin{array}{l}\text { No of devices } \\
\text { used }\end{array}$ & Electrodes positioning ${ }^{a}$ & $\begin{array}{l}\text { Electrode } \\
\text { surface }\left(\mathrm{cm}^{2}\right)\end{array}$ & I (mA) & $t$ (min) & $\begin{array}{l}\text { No of sessions } \\
\text { (n/day) }\end{array}$ \\
\hline Brunelin et $\mathrm{al}^{20}$ & $\begin{array}{l}\text { Neuroconn eldith } \\
\text { DC stimulator plus }\end{array}$ & 1 & $\begin{array}{l}\text { A: midpoint between } \mathrm{F} 3 \text { and } \mathrm{FPI} \text {; } \\
\text { C: midpoint between } \mathrm{T} 3 \text { and } \mathrm{P} 3 \\
\text { Bilateral stimulation } \mathrm{A}: \mathrm{F} 3 \text { and } \mathrm{F} 4 \\
\mathrm{C} \text { : } \mathrm{TP} 3 \text { and TP4 }\end{array}$ & 35 & 2 & 20 & $10(2 \times /$ day $)$ \\
\hline Fitzgerald et $\mathrm{al}^{23}$ & $\begin{array}{l}\text { Neuroconn eldith } \\
\text { DC stimulator plus }\end{array}$ & 1 & Unilateral stimulation A: F3; C: TP3 & 35 & 2 & 20 & $15(I \times /$ day $)$ \\
\hline Mondino et $\mathrm{al}^{25}$ & $\begin{array}{l}\text { Neuroconn eldith } \\
\text { DC stimulator plus }\end{array}$ & 1 & $\begin{array}{l}\mathrm{A} \text { : midpoint between } \mathrm{F} 3 \text { and } \mathrm{FPI} \text {; } \\
\mathrm{C} \text { : midpoint between } \mathrm{T} 3 \text { and } \mathrm{P} 3\end{array}$ & 35 & 2 & 20 & $10(2 \times /$ day $)$ \\
\hline Mondino et $\mathrm{al}^{26}$ & $\begin{array}{l}\text { Neuroconn eldith } \\
\text { DC stimulator plus }\end{array}$ & 1 & $\begin{array}{l}\mathrm{A} \text { : midpoint between } \mathrm{F} 3 \text { and } \mathrm{FPI} \text {; } \\
\mathrm{C} \text { : midpoint between } \mathrm{T} 3 \text { and } \mathrm{P} 3\end{array}$ & 35 & 2 & 20 & $10(2 \times /$ day $)$ \\
\hline Smith et $\mathrm{al}^{27}$ & $\begin{array}{l}\text { Chattanooga ionto } \\
\text { system stimulator }\end{array}$ & 1 & A: F3; C: FP2 & 35 & 2 & 20 & $5(\mathrm{I} \times /$ day $)$ \\
\hline Fröhlich et $\mathrm{al}^{28}$ & $\begin{array}{l}\text { Neuroconn eldith } \\
\text { DC stimulator plus }\end{array}$ & 2 & $\begin{array}{l}\mathrm{A}: \text { between } \mathrm{F} 3 \text { and FPI; } \mathrm{C} \text { : between } \\
\mathrm{T} 3 \text { and } \mathrm{P} 3 \text {; return electrode: } \mathrm{Cz}\end{array}$ & 35 & 2 & 20 & $5(\mathrm{I} \times /$ day $)$ \\
\hline
\end{tabular}

Note: apositioning of all electrodes was in accordance with the 10-20 EEG International System.

Abbreviations: I, current intensity; $n$, number; $t$, time of duration of each tDCS session; tDCS, transcranial direct current stimulation. 
and unilateral tDCS (anode over F3 and cathode on TP3; $\mathrm{n}=13) .{ }^{24}$ Stimulation parameters were kept the same for both experiments: 15 sessions distributed throughout three consecutive weeks, $1 \times /$ day, with a voltage of $2 \mathrm{~mA}$ for 20 minutes/session. Both groups had no significant effects on AVH or other psychotic symptoms.

Patients with refractory AVH were enrolled in a randomized, double-blind, parallel trial performed by Mondino et al..$^{25}$ In that trial, 15 of 28 subjects were ex-participants of a previous study conducted by the same group. ${ }^{20}$ Anode electrode was positioned over DLPFC and cathode on TPJ. Individuals underwent twice-daily stimulation sessions for five consecutive days, $2 \times$ /day with a current intensity of $2 \mathrm{~mA}$ for 20 minutes/ session. Greater AVH frequency reduction was observed (mean: $-46 \%$ vs $+7.5 \%$ ) after active versus sham tDCS.

Another randomized, double-blind, parallel group, sham-controlled trial evaluated the effects of tDCS over the left TPJ in a rest state. ${ }^{26}$ Schizophrenia subjects with drugresistant AVH $(n=23)$ were randomly allocated to active $(\mathrm{n}=11)$ and sham interventions $(\mathrm{n}=12)$. Anodal stimulation was delivered on DLPFC and cathodal tDCS over TPJ. Stimulation sessions were performed for five consecutive days, $2 \mathrm{~mA}$ for 20 minutes/session. The primary outcome measure for AVH was the AHRS scale that was applied before the beginning of the 1st session and following the 10th session. ${ }^{21}$ Greater AVH reduction (mean AHRS: $-28 \%$ vs $-10 \%$ ) following active tDCS was observed, when compared with sham intervention.

In a trial, the effects of tDCS on cognition, psychiatric symptoms and fissure due to cigarettes were evaluated in smoker schizophrenic patients. ${ }^{27}$ Thirty-three patients with schizophrenia or schizoaffective disorder were randomly allocated to the active $(n=17)$ and sham groups $(n=16)$, but only 29 of them completed the entire study. Stimulation sessions were performed for five consecutive days, $2 \times /$ day (anode over DLPFC; cathode on TPJ). Despite improvements in cognitive measures, no difference in PANSS scores, AVH, and cigarette craving was observed after active stimulation.

Fröhlich et al in a parallel, randomized, double-blind, sham-controlled trial evaluated the effects of tDCS applied once a day, on the severity of AVHs in 26 schizophrenic or schizoaffective subjects. ${ }^{28}$ The stimulation parameters were $2 \mathrm{~mA}$ (anodal and cathodal) applied at the same time for 20 minutes/session/day for five consecutive days. ${ }^{28}$ Anodal tDCS was delivered over DLPFC and cathodal on TPJ. Following the active stimulation, no difference in AVH reduction (mean AHRS: $-24 \%$ vs $-34 \%$ ) was observed, when compared with sham group.

\section{Tolerability and adverse events of tDCS}

All patients included in the six randomized clinical trials $(n=164)$ underwent at least one session of tDCS. Of these, a total of 160 completed all sessions and evaluations foreseen. Four individuals, who did not complete the stimulation protocols fully in one of the studies, dropped off for reasons of desisting. ${ }^{27}$ Thus, no study needed to exclude any patient from a certain stimulation protocol as a result of adverse events. ${ }^{20,23,25-28}$

With the exception of two studies, which did not provide information on tolerability in their publications, adverse events secondary to tDCS were investigated by means of questionnaires that were applied at the end of each session. ${ }^{25-28}$ Another two studies also published the adverse events most common in their stimulation protocols, but did not specify the instrument used to obtain the tolerability data..$^{20,23}$

\section{Discussion}

Auditory hallucinations may be considered the main symptom of schizophrenia, due to their high prevalence during the course of the disease in the population of patients. The treatment at present recommended by the American Psychiatric Association (APA) is mainly based on the use of antipsychotic drugs that sometimes may not guarantee an efficient therapeutic response to the reduction of these symptoms. ${ }^{29}$ The daily persistence of hallucinatory experiences is related to compromise of the quality of life and also to socio-occupational damage to the individual's life. In this challenging context, tDCS appears as an emergent treatment for the management of auditory hallucinations in schizophrenia, by means of the phenomenon of neuromodulation.

The present systematic review identified six randomized clinical trials, all at the experimental phase, which sought to evaluate the therapeutic effects of tDCS on auditory hallucinations in schizophrenia, and improvement in the stimulation parameters applied in this context. Analysis of the clinical results of these studies pointed toward incongruence in the information with regard to the therapeutic use of tDCS with a view to reducing the severity of auditory hallucinations in schizophrenia. Only three studies demonstrated a therapeutic benefit of tDCS for the treatment of AVHs, manifested by reductions in severity and frequency of these symptoms in schizophrenic patients. ${ }^{20,25,26}$ Brunelin et al also revealed maintenance of tDCS acute effects on the AHRS scores in the first and third months of follow-up, after 10 sessions of tDCS ( $2 \times /$ day $).{ }^{20}$ However, three other studies were unable to reproduce similar effects on the severity of AVHs. ${ }^{23,27,28}$ 
Division of the clinical trials according to the therapeutic results of tDCS they presented (positive vs negative) also revealed another difference between these two groups: the baseline diagnosis of the individuals evaluated. Trials that demonstrated the therapeutic success of tDCS included only schizophrenic patients in their samples, whereas other studies also included schizoaffective individuals. Nevertheless, the therapeutic success of tDCS on auditory hallucinations cannot be justified by the mental disturbance because the inclusion of individuals in each trial required the fulfillment of eligibility criteria, thereby avoiding possible clinical differences among subjects.

All studies evaluating the therapeutic efficacy of tDCS on AVH adopted as inclusion criterion schizophrenic patients with AVH refractory to treatment, with exception of the study by Smith et al, which may also explain the discrete changes observed in the severity of hallucinations post interventions, in this study. ${ }^{20,23,25-28}$ These findings may also be related to aspects of the sample population. Nicotine-related smoking inclusion criteria of the schizophrenic patients included in this experiment - is associated with a reduction in the clinical efficacy of tDCS in individuals with schizophrenia. ${ }^{30}$ Another important factor that appears to have contributed to the negative response presented in this study was the montage used. Cathodal electrode was placed over the contralateral supraorbital area, different from the other studies that applied cathodal stimulation on TPJ. Neurophysiological studies have shown hyperactivity in left parietal areas of patients with schizophrenia, which is associated with the presence of positive symptoms. ${ }^{18,19,31-34}$ Therefore, the possible reduction of excitability in this area in addition to anodal stimulation on DLPFC may result in a better control of symptoms.

Different definitions of refractoriness were adopted for composition of the inclusion criteria of each study. Fitzgerald et al and Fröhlich et al were the only researchers who followed the refractoriness criteria proposed by the APA for refractory schizophrenia, although the evaluation of AVHs severity of their samples differed. ${ }^{23,28,29}$ Fitzgerald et al used the PANSS scale, what may explain the lack of therapeutic response to tDCS, in this study. The PANSS scale is unable to measure the severity of hallucinations in a detailed manner, and its severity scores for the item of hallucinations are difficult to differentiate from one another. ${ }^{35}$ The more appropriate scale for population samples containing schizophrenic patients with hallucinations refractory to treatment is the AHRS scale, as it presents a high capacity for investigating the hallucinatory symptoms experienced by the individual. ${ }^{21}$ This was the method applied by Fröhlich et al,
Brunelin et al and Mondino et al for quantifying the severity of AVH, which in these samples was shown to be of similar dimensions. ${ }^{20,26,28}$ However, the lack of therapeutic efficacy of tDCS on the AVH described by Fröhlich et al, a result contrary to that obtained by the other two trials, reveals the significant importance of adopting an adequate stimulation protocol for obtaining successful therapy in a population with the potential for symptomatic improvement by means of tDCS. ${ }^{20,26,28}$ Although this trial applied the same current density observed in other studies presenting positive findings, the number of sessions and montage was different. Previous studies revealed better and longer-lasting modulatory response when applying a greater number of sessions and/or a shorter interval among them..$^{20,25}$ The same cannot be affirmed regarding montage, due to still controversial findings. A recent study has even questioned the polarity-specific effects when different current intensities are applied. ${ }^{36}$ Despite an effort having been made to standardize the application of tDCS in different neuropsychiatric and pain conditions, to date there is no established definition as to the optimal protocol in any of these nosological conditions. In addition, another explanation suggested by the authors regarding the non-significant findings observed in their clinical trial is based on the occurrence of a possible placebo effect because all subjects were aware that a new and potentially effective therapy would be investigated in that study. Furthermore, patients were on continuous therapy during the trial with different antipsychotics, some still in combination with other psychotropic drugs.

Although the ideal parameters of tDCS are as yet not completely established in the literature, few but relevant differences were found between the stimulation protocols applied by the clinical trials included in this review, as previously mentioned. The studies that demonstrated a therapeutic benefit of tDCS on AVH refractory to treatment with antipsychotic agents in schizophrenic patients presented identical stimulation parameters. ${ }^{20,25,26}$ All the studies performed anodal stimulation on the left DLPFC with a view to increasing the cortical excitability in this area, since schizophrenic patients possibly present a cortical hypoactivity in this region during the state of rest or when they are submitted to working memory (WM) tasks..$^{37,38}$ Prefrontal cortex dysfunction is associated with development of negative symptoms and compromise of cognitive functions in schizophrenia. ${ }^{17}$ The use of high-frequency repetitive transcranial magnetic stimulation (rTMS), a non-invasive brain stimulation technique that applies magnetic field to modulate brain excitability, for the treatment of negative symptoms 
encouraged the first tDCS studies to be conducted with a view to modulate DLPFC. ${ }^{39}$ The recent discovery that anodal stimulation could improve the performance of working memory, a function involved in the executive control of information, generated speculations about the possibility of left DLPFC stimulation helping to control AVH by inhibiting irrelevant verbal information. ${ }^{17,40}$ In addition, the placement of cathodal electrode over the left parietal area possibly plays an important role as it may decrease the hyperactivity of this area, which is a physiological mechanism associated with positive symptoms in this population, as commented before. ${ }^{18,19,31-34}$ Studies using functional neuroimaging methods have demonstrated that hyperactivity of the left TPJ plays a crucial role in the physiopathology of auditory hallucinations, and it is therefore a target region of cathodal stimulation in all randomized clinical trials that presented AVH as the primary outcome. ${ }^{18-20,23,25,26,28}$ Those trials were motivated by the positive results of studies involving lowfrequency rTMS on the left TPJ, which revealed an attenuation of auditory hallucinations refractory to treatment with antipsychotic drugs. ${ }^{41,42}$

The studies of Brunelin et al and Mondino et al were the only ones of this review that exhibited significant results of both anodal stimulation over DLPFC and cathodal stimulation on left TPJ, which strengthen the hypothesis of the importance of these areas for the control of AVH in refractory patients. ${ }^{17,20,26}$ Another study from the same group of researchers has demonstrated only the efficacy of cathodal stimulation with TPJ as the target region for promoting changes in the severity of AVH. ${ }^{25}$ The absence of therapeutic response to negative symptoms of the patients analyzed may be explained by the unavailability of the PANSS scale data at the end of the last tDCS session, or due to the fact that the study did not present strict eligibility criteria, compromising the quality of the sample. ${ }^{25}$ Moreover, the use of a scale based only on the evaluation of the frequency of $\mathrm{AVH}$, in which the evaluation criteria of the score and the reasons for the use of this method by the authors were not mentioned, may have produced errors in the evaluation of the AVH, such as lack of precision. ${ }^{25}$

The studies of Fitzgerald et al, Smith et al and Fröhlich et al presented a total number of daily tDCS sessions differing from those of previous studies, as previously mentioned. ${ }^{20,23,25-28}$ Stimulation of the motor cortex with tDCS is dependent on a complex maintenance of its effects that are capable of exceeding the period of time of the sessions. ${ }^{43}$ Therefore, stimulation protocols containing only one daily session do not appear to be ideal when the aim is to obtain a therapeutic effect. Moreover, studies with rTMS have recommended treatments of longer duration (around 4-6 weeks) for depressive disorders. ${ }^{44}$ Therefore, the need for conducting further studies in this area becomes evident, with a view to establishing the ideal tDCS protocol.

Unfortunately, not all studies provide data on antipsychotic use, which is a very important issue to discuss as potential moderator of the therapeutic effect of tDCS. In a recent study, Agarwal et al assessed the influence of the type of antipsychotic drug in tDCS effects in individuals with schizophrenia, finding a significant effect in the control of auditory hallucinations, mainly among individuals using low-affinity antipsychotics or a combination of it with highaffinity ones. ${ }^{45}$ Regarding the trials included in this review, although some of them correctly enrolled only patients with stable antipsychotic dose for a minimum period of time, for example 3 months, some relevant information such as type of drug and dose was not present and also was not analyzed by most of them.

All of the tDCS interventions evaluated presented recommended safety measures: electrical current $<2.5 \mathrm{~mA}$, duration of session between 20 and 60 minutes, frequency of sessions $\leq 2$ daily sessions, and electrodes that did not come into contact with the skin. ${ }^{23,25-28,30,46}$ The adverse events frequently related in studies such as itching in the location of electrode, headache, and tingling corroborate pre-existent data in the literature. ${ }^{47}$ No stimulation protocol analyzed needed to be interrupted, thus strengthening the safety profile of this technique. ${ }^{48}$

\section{Conclusion}

tDCS - a safe, portable, and simple neuromodulation technique - has been shown to be promising as a therapeutic approach for auditory hallucinations, particularly the type refractory to treatment with antipsychotic drugs in schizophrenic patients. Nevertheless, only six randomized clinical trials with limited sample sizes have been published and, of these, only three demonstrated therapeutic benefits with the use of this technique. The body of information found in the current literature has been shown to be insufficient for determining the effectiveness of treatment with tDCS for reducing the severity of auditory hallucinations. Randomized clinical trials containing large population samples are expected to investigate whether the positive results can be replicated. Moreover, the stimulation parameters and methods of evaluating the clinical outcomes need to be better defined, so that there will be advances in the sense of consolidating tDCS as a therapeutic possibility for auditory hallucinations in schizophrenia. 


\section{Disclosure}

The authors report no conflicts of interest in this work.

\section{References}

1. Kuo MF, Paulus W, Nitsche MA. Therapeutic effects of non-invasive brain stimulation with direct currents (tDCS) in neuropsychiatric diseases. Neuroimage. 2014;85(Pt 3):948-960.

2. Frangou S. Schizophrenia. Medicine. 2008;36(8):405-409.

3. McGrath J, Saha S, Chant D, Welham J. Schizophrenia: a concise overview of incidence, prevalence, and mortality. Epidemiol Rev. 2008; 30:67-76.

4. Picchioni MM, Murray RM. Schizophrenia. BMJ. 2007;335(7610): 91-95.

5. Howes OD, Murray RM. Schizophrenia: an integrated sociodevelopmental-cognitive model. Lancet. 2014;383(9929):1677-1687.

6. David AS. The cognitive neuropsychiatry of auditory verbal hallucinations: an overview. Cogn Neuropsychiatry. 2004;9(1-2):107-123.

7. American Psychiatric Association. Schizophrenia spectrum and other psychotic disorders. In: Diagnostic and Statistical Manual of Mental Disorders. 5th ed. Washington DC, MD: American Psychiatric Publishing; 2013:87-122.

8. Andreasen NC, Flaum M. Schizophrenia: the characteristic symptoms. Schizophr Bull. 1991;17(1):27-49.

9. Kubera KM, Barth A, Hirjak D, Thomann PA, Wolf RC. Noninvasive brain stimulation for the treatment of auditory verbal hallucinations in schizophrenia: methods, effects and challenges. Front Syst Neurosci. 2015;9:131.

10. Harkavy-Friedman JM, Kimhy D, Nelson EA, Venarde DF, Malaspina D, Mann JJ. Suicide attempts in schizophrenia: the role of command auditory hallucinations for suicide. J Clin Psychiatry. 2003;64(8):871-874.

11. McCarthy-Jones S, Trauer T, Mackinnon A, Sims E, Thomas N, Copolov DL. A new phenomenological survey of auditory hallucinations: evidence for subtypes and implications for theory and practice. Schizophr Bull. 2014;40(1):231-235.

12. Dayan E, Censor N, Buch ER, Sandrini M, Cohen LG. Noninvasive brain stimulation: from physiology to network dynamics and back. Nat Neurosci. 2013;16(7):838-844.

13. Wagner T, Valero-Cabre A, Pascual-Leone A. Noninvasive human brain stimulation. Annu Rev Biomed Eng. 2007;9:527-565.

14. Nitsche MA, Cohen LG, Wassermann EM, et al. Transcranial direct current stimulation: state of the art 2008. Brain Stimul. 2008;1(3):206-223.

15. Koops S, van den Brink H, Sommer IE. Transcranial direct current stimulation as a treatment for auditory hallucinations. Front Psychol. 2015;6:244.

16. Agarwal SM, Shivakumar V, Bose A, et al. Transcranial direct current stimulation in schizophrenia. Clin Psychopharmacol Neurosci. 2013; 11(3):118-125.

17. Brunoni AR, Shiozawa $P$, Truong D, et al. Understanding tDCS effects in schizophrenia: a systematic review of clinical data and an integrated computation modeling analysis. Expert Rev Med Devices. 2014;11(4): 383-394.

18. Silbersweig DA, Stern E, Frith C, et al. A functional neuroanatomy of hallucinations in schizophrenia. Nature. 1995;378(6553):176-179.

19. Shergill SS, Brammer MJ, Williams SC, Murray RM, McGuire PK. Mapping auditory hallucinations in schizophrenia using functional magnetic resonance imaging. Arch Gen Psychiatry. 2000;57(11): 1033-1038.

20. Brunelin J, Mondino M, Gassab L, et al. Examining transcranial direct-current stimulation (tDCS) as a treatment for hallucinations in schizophrenia. Am J Psychiatry. 2012;169(7):719-724.

21. Haddock G, McCarron J, Tarrier N, Faragher EB. Scales to measure dimensions of hallucinations and delusions: the Psychotic Symptom Rating Scales (PSYRATS). Psychol Med. 1999;29(4):879-889.
22. Kay SR, Fiszbein A, Opler LA. The Positive and Negative Syndrome Scale (PANSS) for schizophrenia. Schizophrenia Bull. 1987;13(2):261-276.

23. Fitzgerald PB, McQueen S, Daskalakis ZJ, Hoy KE. A negative pilot study of daily bimodal transcranial direct current stimulation in schizophrenia. Brain Stimul. 2014;7(6):813-816.

24. Sheehan DV, Lecrubier Y, Sheehan KH, et al. The mini-international neuropsychiatric interview (M.I.N.I.): the development and validation of a structured diagnostic psychiatric interview for DSM-IV and ICD-10. J Clin Psychiatry. 1998;59(Suppl 20):22-33.

25. Mondino M, Haesebaert F, Poulet E, Suaud-Chagny MF, Brunelin J. Fronto-temporal transcranial direct current stimulation (tDCS) reduces source-monitoring deficits and auditory hallucinations in patients with schizophrenia. Schizophr Res. 2015;161(2-3):515-516.

26. Mondino M, Jardri R, Suaud-Chagny MF, Saoud M, Poulet E, Brunelin J. Effects of fronto-temporal transcranial direct current stimulation on auditory verbal hallucinations and resting-state functional connectivity of the left temporo-parietal junction in patients with schizophrenia. Schizophr Bull. 2016;42(2):318-326.

27. Smith RC, Boules S, Mattiuz S, et al. Effects of transcranial direct current stimulation (tDCS) on cognition, symptoms, and smoking in schizophrenia: a randomized controlled study. Schizophr Res. 2015;168(1-2): 260-266.

28. Fröhlich F, Burrello TN, Mellin JM, et al. Exploratory study of once-daily transcranial direct current stimulation (tDCS) as a treatment for auditory hallucinations in schizophrenia. Eur Psychiatry. 2016;33:54-60.

29. Lehman AF, Lieberman JA, Dixon LB, et al; American Psychiatric Association, Steering Committee on Practice Guidelines. Practice guideline for the treatment of patients with schizophrenia, second edition. Am J Psychiatry. 2004;161(Suppl 2):1-56.

30. Brunelin J, Hasan A, Haesebaert F, Nitsche MA, Poulet E. Nicotine smoking prevents the effects of frontotemporal transcranial direct current stimulation (tDCS) in hallucinating patients with schizophrenia. Brain Stimul. 2015;8(6):1225-1227.

31. Wible CG, Preus AP, Hashimoto R. A cognitive neuroscience view of schizophrenic symptoms: abnormal activation of a system for social perception and communication. Brain Imaging Behav. 2009;3(1): $85-110$.

32. Rains LS, Fallica G, O'Daly O, et al. Exploring psychotic symptoms: a comparison of motor related neuronal activation during and after acute psychosis. BMC Psychiatry. 2012;12:102.

33. Molina V, Reig S, Pascau J, et al. Anatomical and functional cerebral variables associated with basal symptoms but not risperidone response in minimally treated schizophrenia. Psychiatry Res. 2003;124(3): 163-175.

34. Franck N, O'Leary DS, Flaum M, Hichwa RD, Andreasen NC. Cerebral blood flow changes associated with Schneiderian first-rank symptoms in schizophrenia. J Neuropsychiatry Clin Neurosci. 2002;14(3): 277-282.

35. Santor DA, Ascher-Svanum H, Lindenmayer JP, Obenchain RL. Item response analysis of the Positive and Negative Syndrome Scale. BMC Psychiatry. 2007;7:66.

36. Batsikadze G, Moliadze V, Paulus W, Kuo MF, Nitsche MA. Partially non-linear stimulation intensity-dependent effects of direct current stimulation on motor cortex excitability in humans. J Physiol. 2013;591(7): $1987-2000$

37. Kuhn S, Gallinat J. Resting-state brain activity in schizophrenia and major depression: a quantitative meta-analysis. Schizophr Bull. 2013; 39(2):358-365.

38. Lawrie SM, Buechel C, Whalley HC, Frith CD, Friston KJ, Johnstone EC. Reduced frontotemporal functional connectivity in schizophrenia associated with auditory hallucinations. Biol Psychiatry. 2002;51(12): 1008-1011.

39. Dlabac-de Lange JJ, Knegtering R, Aleman A. Repetitive transcranial magnetic stimulation for negative symptoms of schizophrenia: review and meta-analysis. J Clin Psychiatry. 2010;71(4):411-418. 
40. Fregni F, Boggio PS, Nitsche M, et al. Anodal transcranial direct current stimulation of prefrontal cortex enhances working memory. Exp Brain Res. 2005;166(1):23-30.

41. Aleman A, Sommer IE, Kahn RS. Efficacy of slow repetitive transcrania magnetic stimulation in the treatment of resistant auditory hallucinations in schizophrenia: a meta-analysis. J Clin Psychiatry. 2007;68(3): 416-421.

42. Montagne-Larmurier A, Etard O, Maiza O, Dollfus S. Repetitive transcranial magnetic stimulation in the treatment of auditory hallucinations in schizophrenic patients. Curr Opin Psychiatry. 2011;24(6): 533-540.

43. Monte-Silva K, Kuo MF, Liebetanz D, Paulus W, Nitsche MA. Shaping the optimal repetition interval for cathodal transcranial direct current stimulation (tDCS). J Neurophysiol. 2010;103(4):1735-1740.

44. Carpenter LL, Janicak PG, Aaronson ST, et al. Transcranial magnetic stimulation (TMS) for major depression: a multisite, naturalistic, observational study of acute treatment outcomes in clinical practice. Depress Anxiety. 2012;29(7):587-596.
45. Agarwal SM, Bose A, Shivakumar V, et al. Impact of antipsychotic medication on transcranial direct current stimulation (tDCS) effects in schizophrenia patients. Psychiatry Res. 2016;235:97-103.

46. Fregni F, Nitsche MA, Loo CK, et al. Regulatory considerations for the clinical and research use of transcranial direct current stimulation (tDCS): review and recommendations from an expert panel. Clin Res Regul Aff. 2015;32(1):22-35.

47. Poreisz C, Boros K, Antal A, Paulus W. Safety aspects of transcranial direct current stimulation concerning healthy subjects and patients. Brain Res Bull. 2007;72(4-6):208-214.

48. Rossi S, Hallett M, Rossini PM, Pascual-Leone A; Safety of TMS Consensus Group. Safety, ethical considerations, and application guidelines for the use of transcranial magnetic stimulation in clinical practice and research. Clin Neurophysiol. 2009;120(12):2008-2039.
Neuropsychiatric Disease and Treatment

\section{Publish your work in this journal}

Neuropsychiatric Disease and Treatment is an international, peerreviewed journal of clinical therapeutics and pharmacology focusing on concise rapid reporting of clinical or pre-clinical studies on a range of neuropsychiatric and neurological disorders. This journal is indexed on PubMed Central, the 'PsycINFO' database and CAS,

\section{Dovepress}

and is the official journal of The International Neuropsychiatric Association (INA). The manuscript management system is completely online and includes a very quick and fair peer-review system, which is all easy to use. Visit http://www.dovepress.com/testimonials.php to read real quotes from published authors.

Submit your manuscript here: http://www.dovepress.com/neuropsychiatric-disease-and-treatment-journal 